\title{
Accurate Electronic, Transport, and Related Properties of Wurtzite Beryllium Oxide (w-Be0)
}

\author{
Cheick Oumar Bamba1, Richard Inakpenu' ${ }^{1}$ Yacouba I. Diakite², Lashounda Franklin'1, \\ Yuriy Malozovsky', Anthony D. Stewart' ${ }^{1}$, Diola Bagayoko' ${ }^{1}$ \\ ${ }^{1}$ Department of Physics, Southern University and A\&M College, Baton Rouge, LA, USA \\ ${ }^{2}$ Department of Physics, University of Sciences, Techniques and Technologies of Bamako, Bamako, Mali \\ Email: bagayoko@aol.com
}

How to cite this paper: Bamba, C.O., Inakpenu, R., Diakite, Y.I., Franklin, L., Malozovsky, Y., Stewart, A.D. and Bagayoko, D. (2017) Accurate Electronic, Transport, and Related Properties of Wurtzite Beryllium Oxide (w-BeO). Journal of Modern Physics, 8, 1938-1949.

https://doi.org/10.4236/jmp.2017.812116

Received: September 21, 2017

Accepted: November 14, 2017

Published: November 17, 2017

Copyright (c) 2017 by authors and Scientific Research Publishing Inc. This work is licensed under the Creative Commons Attribution International License (CC BY 4.0).

http://creativecommons.org/licenses/by/4.0/

(c) (i) Open Access

\begin{abstract}
We report details of our ab-initio, self-consistent density functional theory (DFT) calculations of electronic and related properties of wurtzite beryllium oxide ( $w-B e O)$. Our calculations were performed using a local density approximation (LDA) potential and the linear combination of atomic orbitals (LCAO) formalism. Unlike previous DFT studies of $\mathrm{BeO}$, the implementation of the Bagayoko, Zhao, and Williams (BZW) method, as enhanced by the work of Ekuma and Franklin (BZW-EF), ensures the full physical content of the results of our calculations, as per the derivation of DFT. We present our computed band gap, total and partial densities of states, and effective masses. Our direct band gap of $10.30 \mathrm{eV}$, reached by using the experimental lattice constants of $\mathrm{a}=2.6979 \AA$ and $\mathrm{c}=4.3772 \AA$ at room temperature, agrees very well the experimental values of $10.28 \mathrm{eV}$ and $10.3 \mathrm{eV}$. The hybridization of $\mathrm{O}$ and Be $\mathrm{p}$ states in the upper valence bands, as per our calculated, partial densities of states, are in agreement with corresponding, experimental findings.
\end{abstract}

\section{Keywords}

Density Functional Theory, BZW-EF Method, Accurate, Calculated Properties

\section{Introduction}

Beryllium oxide, also known as beryllia, has attracted much interest due to its current and potential applications. Second only to diamond among electrically insulating materials, $\mathrm{BeO}$ is an excellent thermal conductor with a measured room temperature band gap of as high as an estimated $10.63 \mathrm{eV}[1]$ and as low as 
$10.28 \mathrm{eV}$ [2] and a relatively low dielectric constant. These properties make it an ideal candidate for next generation dielectrics in high performance, semiconductor devices. $\mathrm{BeO}$ is also a mechanically robust material that can be used in transparent coatings for telescope mirrors. Owing to its sensitivity in the Schumann region of the electromagnetic spectrum and resistance to radiation, $\mathrm{BeO}$ can be used for electronics in low-earth orbiting (LEO) and outer space applications to monitor global events from lightning activity and earthquake prediction to variations of water vapor in the atmosphere which may impact global temperatures.

Several sources have reported results of experimental studies of $\mathrm{w}-\mathrm{BeO}$ as indicated in Table 1. C. Peterson et al. [3] measured the transmittance of a single-crystal $\mathrm{BeO}$ at $300 \mathrm{~K}$, they observed zero transmittance at $10.3 \mathrm{eV}$. Bellamy et al. [4] used precise X-ray methods in their measurements. The lattice parameters of the sample were determined, at $21^{\circ} \mathrm{C}$, more accurately using a least squares method; they reported values of $\mathrm{a}=2.6979 \pm 0.001 \AA$ and $\mathrm{c}=4.3772 \pm 0.0002 \AA$. The group led by Roessler [1] employed normal-incidence reflectance on a single crystal of $\mathrm{w}-\mathrm{BeO}$. They made their reflectance measurements at $300 \mathrm{~K}$ and near-normal incidence. They found that $\mathrm{BeO}$ has a wurtzite structure with a hexagonal prismatic Brillouin zone and a direct band gap estimated to be $10.63 \pm$ $0.10 \mathrm{eV}$. Measurement of the polarization-dependent reflectance spectra of $\mathrm{BeO}$ for phonon energies $0.6<\hbar \omega<30$ was performed by Freeouf [2] using a synchrotron radiation and standard light sources and obtained a gap of $10.30 \mathrm{eV}$. An energy gap of $10.28 \mathrm{eV}$ was obtained for the electric field parallel and perpendicular to the $c$ axis. Grundler and co-workers [5] performed energy loss measurements on amorphous and polycrystalline $\mathrm{BeO}$ thin films, applying 25 $\mathrm{KeV}$ electrons, and reported a band gap of $9.6 \mathrm{eV}$; we note that this result does not pertain to a bulk, single crystal $\mathrm{w}-\mathrm{BeO}$, the system under study here. The discrepancies between the experimental values reported for $\mathrm{w}-\mathrm{BeO}$, ranging from 10.28 to $10.63 \mathrm{eV}$, are likely due to the differences in growth conditions and the related quality of the samples and uncertainties associated with measurement techniques.

While the band gap is somewhat established experimentally, previous theoretical calculations reported band gaps over a wide range. The calculated values disagree among themselves and with experiment as can be seen in Table 2. For

Table 1. Measured band gaps (Eg in $\mathrm{eV}$ ) of wurtzite beryllium oxide at room temperature.

\begin{tabular}{cc}
\hline Experimental works & $\mathbf{E g}(\mathrm{eV})$ \\
\hline $\begin{array}{c}\text { Optical absorption edge on single crystal BeO at 300 K } \\
\text { Normal-incidence reflectance of wurtzite BeO at room } \\
\text { temperature (estimated band gap value) }\end{array}$ & $10.3^{3}$ \\
Far-ultraviolet reflectance at room temperature & $10.63 \pm 0.10^{1}$ \\
Electron energy loss measurements for amorphous and \\
polycrystalline BeO thin films
\end{tabular}


Table 2. Previous calculated, band gaps (Eg in $\mathrm{eV}$ ) of $\mathrm{w}-\mathrm{BeO}$ reported in the literature.

\begin{tabular}{ccc}
\hline Computational method & Potentials (DFT and Other) & Eg (eV) \\
\hline Non local pseudopotential & LDA & $7.0^{\mathrm{a}}$ \\
LMTO-ASA and PAW & LDA & $7.23^{\mathrm{b}}$ \\
Nonlocal, norm-conserving pseudopotential & LDA & $7.36^{\mathrm{c}}$ \\
Plane wave pseudopotential & LDA & $7.36^{\mathrm{d}}$ \\
SIC-pseudopotential & LDA & $7.41^{\mathrm{e}}$ \\
Plane wave pseudopotential & LDA & $7.42^{\mathrm{f}}$ \\
OLCAO & LDA & $7.54^{\mathrm{g}}$ \\
Nonlocal empirical pseudopotential & LDA & $7.63^{\mathrm{h}}$ \\
Ab initio pseudopotential & LDA & $7.8^{\mathrm{i}}$ \\
Plane wave basis method & GGA & $6.99^{\mathrm{j}}$ \\
FP-LAPW + lo & GGA & $7.44^{\mathrm{k}}$ \\
FP-LAPW + lo & GGA & $7.47^{\mathrm{l}}$ \\
Pseudopotential-PAW & GGA & $7.48^{\mathrm{m}}$ \\
Plane wave pseudopotential & GGA & $7.50^{\mathrm{h}}$ \\
Plane wave pseudopotential & GGA & $7.66^{\mathrm{o}}$ \\
Nonlocal empirical pseudopotential & GGA & $7.70^{\mathrm{h}}$ \\
PP + LAPW + lo & GGA & $8.4^{\mathrm{p}}$ \\
FP-LAPW + lo & GGA & $8.4^{\mathrm{q}}$ \\
LMTO-ASA & GGA & $8.57^{\mathrm{k}}$ \\
\hline
\end{tabular}

${ }^{\mathrm{a} R e f e r e n c e ~[6] ; ~}{ }^{\mathrm{b}}$ Reference [7]; 'Reference [8]; ${ }^{\mathrm{A}}$ Reference [9]; ${ }^{\mathrm{e}}$ Reference [8]; ${ }^{\mathrm{R}}$ Reference [10]; ${ }^{\mathrm{B}}$ Reference [11]; ${ }^{\mathrm{h}}$ Reference [17]; ${ }^{\mathrm{i}}$ Reference [12]; ${ }^{\mathrm{j}}$ Reference [13]; ${ }^{\mathrm{k}}$ Reference [20]; ${ }^{\mathrm{m}}$ Reference [14]; ${ }^{\mathrm{n}}$ Reference [15]; ${ }^{\circ}$ Reference [16]; ${ }^{\mathrm{P}}$ Reference [18]; ${ }^{\mathrm{q}}$ Reference [19]; ${ }^{\mathrm{r}}$ Reference [21]; ${ }^{\mathrm{s}}$ Reference [22].

ab-initio LDA potential [6]-[12], the calculated band gap of $\mathrm{w}-\mathrm{BeO}$ is between $7.0 \mathrm{eV}$ and $7.63 \mathrm{eV}$. Baumeier et al. [8] employed norm-conserving pseudopotentials and self-interaction corrections (SIC) and reach a band gap of $10.5 \mathrm{eV}$. Vydrov [13] executed self-consistently calculations employing the Perdew-Zunger functional and self-interaction corrections (PZ-SIC) and carried out systematic tests of the performance of PZ-SIC; he found that PZ-SIC overcorrects for the band gap of many-electron systems. Previous, ab-initio generalized gradient approximations (GGA) calculations [14]-[21] have reported band gaps ranging from $6.99 \mathrm{eV}$ to $8.4 \mathrm{eV}$. Bouhalouane et al. [21] employed the full-potential linearized augmented plane wave plus local orbitals (FP-LAPW-lo) formalism and reached a band gap value of $8.57 \mathrm{eV}$. The group led by Groh used the Becke's three-parameter hybrid exchange functional (B3LYP) [22] in their DFT calculations and obtained a value of $10.39 \mathrm{eV}$. Makurin et al. [23] utilized the linear muffin-tin orbital method in an atomic sphere approximation (LMTO-ASA) and found $9.5 \mathrm{eV}$ for the band gap of $\mathrm{w}-\mathrm{BeO}$. Results from calculation resorting to SIC corrections [13] [14] or employing DFT potential that cannot be obtained 
as a functional derivative of an exchange-correlation energy [19] [21] [22] do not elucidate the woeful underestimation by ab-initio LDA and GGA calculations, in light of the ad hoc nature of some corrections and the adjustable parameters in potentials.

Given the above theoretical results, listed in Table 2, our motivation in this work is to contribute to the resolution of the long-standing underestimation of the energy gap of $\mathrm{BeO}$ by ab-initio DFT calculations. The current and growing potential applications of beryllium oxide add to this motivation. Indeed, $\mathrm{BeO}$ is a special alkali earth oxide; it crystallizes in wurtzite structure at room temperature while other alkali earth oxides crystallize in rock salt structure at the same temperature [23]-[38]. The ionic bonding in $\mathrm{BeO}$ is reported to have some covalent features. Due to these unusual features, $\mathrm{BeO}$ has low electrical conductivity, a high thermal conductivity, a high melting point and is the hardest material among alkali earth oxides [26]. These characteristics make it interesting for electronic, nuclear, aerospace, and electro-technical applications [39]. We explain in the subsequent section details of our computational method.

\section{Method and Computational Details}

More details about our computational approach are available in previous articles [40]-[49]. We used the Ceperley and Alder LDA potential [50] with the parameterization of Vosko and his group [51], the LCAO and the BZW method, as enhanced by Ekuma and Franklin (BZW-EF) [52]. Both the BZW and BZW-EF employ successive augmentation of the initial, small basis set, by one orbital at a time, to execute several self-consistent calculations. The difference between the BZW and BZW-EF methods is the methodical increase of the basis set. In the BZW method, the basis set is increased by adding orbitals in the order of augmenting, unoccupied energies (in atomic or ionic species). As explained by Bagayoko [41], in systems with two or more atoms, the polarization of $\mathrm{p}, \mathrm{d}$ and $\mathrm{f}$ orbitals has primacy over the spherical symmetry of s orbitals, for the valence electrons. In the BZW-EF method, a basis set is increased in such a way that, for any principal quantum number $n$, the $\mathrm{p}, \mathrm{d}$, and $\mathrm{f}$ orbitals, if applicable, are added before the corresponding s orbital of that quantum number.

We used a computer program originated in the US Department of Energy's Ames Laboratory, in Iowa, to perform non-relativistic calculations. We first performed the LCAO self-consistent calculations of the electronic energy at the atomic or ionic level of the material being investigated. These species are $\mathrm{Be}^{2+}$ and $\mathrm{O}^{2-}$. Our choice was based on the results of preliminary calculations for $\mathrm{w}-\mathrm{BeO}$ using neutral atoms. The preliminary results showed a transfer of approximately two electrons from Be to $\mathrm{O}$. We subsequently performed ab-initio; self-consistent calculations to determine the atomic basis set to be used in the solid-state calculations for $\mathrm{Be}^{2+}$ and $\mathrm{O}^{2-}$.

As per by the BZW-EF method, our self-consistent calculations began with a small basis set that contained the minimum basis set (MBS); the MBS is the one 
just big enough to encompass all the electrons in the system. The basis set of the second calculation is composed of the basis set from Calculation I augmented with an appropriate orbital that represents an excited energy level in the ionic species of the system. Then, we compared numerically and graphically the occupied energies from the first (I) and second (II) calculations, after setting the Fermi level to zero. The comparison showed that some valence bands from Calculation II were lower than their corresponding ones from Calculation I. This is the indication that the basis set from Calculation I is not complete for the DFT description of the system being studied. We performed a third self-consistent calculation with the basis set from Calculation II augmented with an appropriate orbital representing an excited level of the ionic species in the system. Again, the occupied energies from Calculations II and III were compared numerically and graphically. This process continued until a given calculation, i.e. $\mathrm{N}$, was found to have the same occupied energy as the two calculations following it, i.e., $\mathrm{N}+1$ and $\mathrm{N}+2$. The superposition of the occupied energies from three consecutive calculations is the indication that one has reached the absolute minima of the occupied energies (i.e., the ground state), as required by DFT. A third calculation is needed to confirm the attainment of the absolute minima of the valence bands, as opposed to local minima. The calculation with the smallest basis set of the three calculations provided the DFT description of the material under study and its basis set is called the optimal basis set. For w-BeO, the basis of Calculation II gives these absolute minima of the occupied energies and provides the DFT description of the material.

When the ground state is reached, further calculations with basis sets larger than the optimal basis set do not modify the occupied energies. In other words, if the basis set of Calculation $\mathrm{N}$ is the optimal basis set, then Calculation $\mathrm{N}+1$, $\mathrm{N}+2, \mathrm{~N}+3$, etc. produce the same occupied energies. However, as per the Rayleigh theorem [53], calculations with basis sets much larger than the optimal basis set lead to an extra lowering of some unoccupied energies. The BZW-EF ensures the minimization of the occupied energies down to the ground state and avoids this mathematical artifact consisting of unphysical lowering of unoccupied energies.

Let an eigenvalue equation be is solved with two basis sets containing $\mathrm{n}$ and $\mathrm{n}+1$ basis functions, respectively, with the smaller basis set totally included in the larger one. The Rayleigh theorem states that the ordered eigenvalues (from the lowest to the highest) are generate in such a fashion that eigenvalues obtained with $(n+1)$ functions are lower than or equal to their corresponding values obtained with $\mathrm{n}$ functions. This theorem clearly explains the reason very large basis sets containing the optimal one are over-complete for the DFT description of the material being study; these very large basis sets do not change the charge density and the Hamiltonian from their respective values generated with the optimal basis set. With no change in the Hamiltonian, the physics of the problem, a change in eigenvalues (unoccupied ones) is a mathematical artifact and not the expres- 
sion of a physical interaction contained in the Hamiltonian.

Details of our computational method for a replication of our calculations follow. $\mathrm{BeO}$ is an element of the space group $\mathrm{P}_{3} \mathrm{mc}$ and have the hexagonal wurtzite structure [54]. There are two atoms of each kind in the unit cell and they are assigned to the special position $2(\mathrm{~b})$ so that Be atoms occupy the sites $\left(\frac{1}{3}, \frac{2}{3}, 0\right)$ and $\left(\frac{2}{3}, \frac{1}{3}, \frac{1}{2}\right)$ while the oxygen atoms are at $\left(\frac{1}{3}, \frac{2}{3}, z\right)$ and $\left(\frac{2}{3}, \frac{1}{3}, \frac{1}{2}+z\right)$. In our self-consistent calculations, we utilized the experimental lattice constants $\mathrm{a}=$ $2.6979 \AA, c=4.3772$ and $z=0.375 \AA$ at room temperature. Our calculations used Gaussian functions. We employed even-tempered Gaussian functions, with minimum and maximum exponents of 0.3 and $0.5585 \times 10^{5}$ for $\mathrm{Be}^{2+}$ and 0.22 and $0.5254 \times 10^{5}$ for $\mathrm{O}^{2-}$. We utilized a $24 \mathrm{k}$ point mesh, with proper weights, in the irreducible Brillouin zone of the wurtzite structure. The computational error for the valence charge was about 0.00479 for 20 electrons or $2 \times 10^{-4}$ per electron. The self-consistent potentials converged to a difference of $10^{-5}$.

After the attainment of self-consistency with the optimal basis set, we produced the final bands using $141 \mathrm{k}$-points in the Brillouin zone. We subsequently utilized $320 \mathrm{k}$ points for the calculations of the total and partial densities of states. We also obtained effectives masses using fits around the minimum and maxima of the conduction and valence bands, respectively.

\section{Results}

Table 3 below shows the successive calculations performed with the goal of reaching the absolute minima of the occupied energies. The band gap, generally, can decrease or increase before one reaches the optimal basis set.

The DFT description of the electronic properties of $\mathrm{w}-\mathrm{BeO}$ is provided by the band structure in Figure 1 below. These bands were obtained from Calculation II, the one for which the valence bands reach their absolute minima. The resulting, calculated band gap is $10.296 \mathrm{eV}$ or $10.30 \mathrm{eV}$. The extra decreasing of some

Table 3. Successive calculations from the BZW-EF method for $\mathrm{w}-\mathrm{BeO}$ (Calculations I-IV). In these calculations, the lattice constants used are $\mathrm{a}=2.6979 \AA$ and $\mathrm{c}=4.3772 \AA$, at room temperature. Calculation II led to the absolute minima of the occupied energies. The band gaps in Column 5 are direct ones, except the one in the last row, from Calculation 6.

\begin{tabular}{ccccc}
\hline & $\begin{array}{c}\text { Beryllium Be } \\
\text { (valence electrons) }\end{array}$ & $\begin{array}{c}\text { Oxygen } \mathrm{O}^{2-} \\
\text { (valence electrons) }\end{array}$ & $\begin{array}{c}\text { Wave functions } \\
2 \mathrm{Be}^{2+} \& 2 \mathrm{O}^{2-}\end{array}$ & $\begin{array}{c}\text { Band gap }(\Gamma-\Gamma) \\
\text { in } \mathrm{eV}\end{array}$ \\
\hline 1 & $1 \mathrm{~s}^{2} 2 \mathrm{~s}^{0} 2 \mathrm{p}^{0}$ & $2 \mathrm{~s}^{2} 2 \mathrm{p}^{6}$ & 36 & 10.630 \\
2 & $1 \mathrm{~s}^{2} 2 \mathrm{~s}^{0} 2 \mathrm{p}^{0} 3 \mathrm{p}^{0}$ & $2 \mathrm{~s}^{2} 2 \mathrm{p}^{6}$ & 48 & 10.296 \\
3 & $1 \mathrm{~s}^{2} 2 \mathrm{~s}^{0} 2 \mathrm{p}^{0} 3 \mathrm{p}^{0} 4 \mathrm{p}^{0}$ & $2 \mathrm{~s}^{2} 2 \mathrm{p}^{6}$ & 60 & 10.300 \\
4 & $1 \mathrm{~s}^{2} 2 \mathrm{~s}^{0} 2 \mathrm{p}^{0} 3 \mathrm{p}^{0} 4 \mathrm{p}^{0} 4 \mathrm{~s}^{0}$ & $2 \mathrm{~s}^{2} 2 \mathrm{p}^{6}$ & 64 & 9.182 \\
5 & $1 \mathrm{~s}^{2} 2 \mathrm{~s}^{0} 2 \mathrm{p}^{0} 3 \mathrm{p}^{0} 4 \mathrm{p}^{0}$ & $2 \mathrm{~s}^{2} 2 \mathrm{p}^{6} 3 \mathrm{~s}^{0}$ & 64 & 7.58 \\
6 & $1 \mathrm{~s}^{2} 2 \mathrm{~s}^{0} 2 \mathrm{p}^{0} 3 \mathrm{p}^{0} 4 \mathrm{p}^{0}$ & $2 \mathrm{~s}^{2} 2 \mathrm{p}^{6} 3 \mathrm{p}^{0}$ & 72 & 9.388 (Indirect) \\
\hline
\end{tabular}


unoccupied energies, obtained with basis sets containing the optimal one, is explained by the Raleigh theorem.

The total density (DOS) and partial density (pDOS) of states, obtained from the bands from Calculation II (with the optimal basis set) are given in Figure 2 and Figure 3, respectively. The total valence bandwidth is $18.773 \mathrm{eV}$, as derived from the band structure in Figure 1 and the eigenvalues in Table 3. The lower and upper valence bandwidths are respectively $2.41 \mathrm{eV}$ and $6.44 \mathrm{eV}$. For the partial density of states (pDOS), we clearly observed a net predominance of the oxygen $\mathrm{p}(\mathrm{O}-\mathrm{p})$ in the upper valence, hybridized with a small contribution from beryllium $\mathrm{p}$ (Be-p). The lowest valence band is entirely from O-s. Contributions to the conduction band are from both Be $\mathrm{p}$ and $\mathrm{s}$.

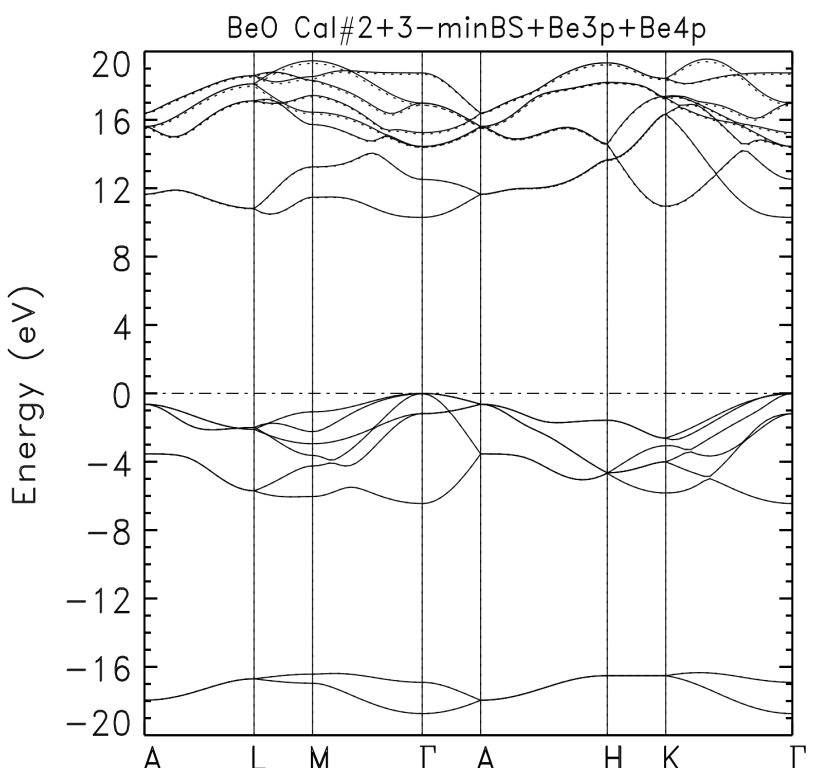

Figure 1. Calculated band structures of wurtzite $\mathrm{BeO}(w-\mathrm{BeO})$, as obtained from Calculation II (full line) and III (dashed line). The calculated energies gap is $10.30 \mathrm{eV}$. The Fermi energy level is set to zero. We note the perfect superposition of the bands from the two calculations $+13 \mathrm{eV}$.

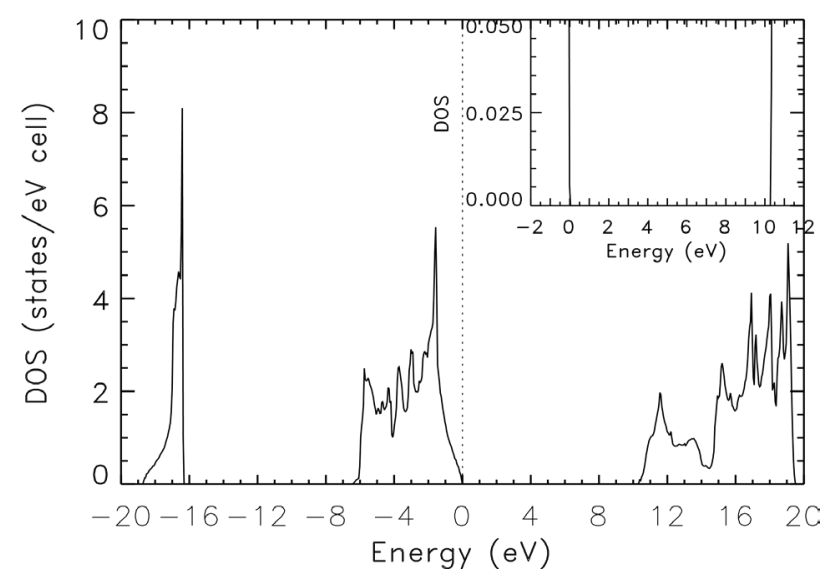

Figure 2. Calculated total density of state (DOS) for w-BeO. 
Although we do not have experimental results for complete comparison, some of our results agree with measurements by Sashin and coworkers [54]. They found that the upper valence band is dominated by the $2 \mathrm{p}$ orbital of $\mathrm{O}$ with a relatively small contribution from the p orbital of Be. However, the bandwidth of their $\mathrm{O}-\mathrm{p}$ is $4.8 \pm 0.3 \mathrm{eV}, 1.64 \mathrm{eV}$ lower than our $6.44 \mathrm{eV}$. The bandwidth of their $\mathrm{O}$-s is $2.1 \pm 0.2 \mathrm{eV}, 0.31 \mathrm{eV}$ lower than our value of $2.4 \mathrm{eV}$. Their total valence bandwidth is $19.4 \pm 0.3 \mathrm{eV}, 0.63 \mathrm{eV}$ larger than our $18.773 \mathrm{eV}$ bandwidth.

We provide, in Table 4 below, a listing of the valence and low-laying conduction band energies at high symmetry points. We expect this table to enable

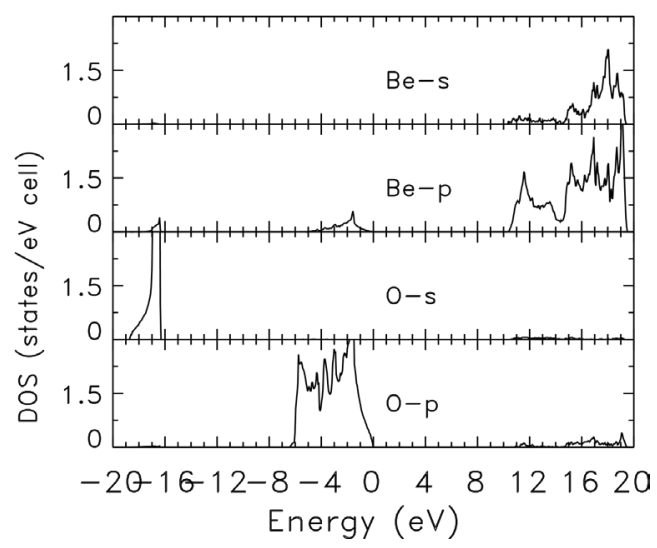

Figure 3. Calculated, partial density of states (p-DOS) as derived from the bands from Calculation II.

Table 4. Electronic energies (in $\mathrm{eV}$ ) of $\mathrm{w}-\mathrm{BeO}$ at the high symmetry points in the Brillouin zone, as obtained from Calculation II. The zero represents the Fermi energy. The calculated, direct band gap is $10.30 \mathrm{eV}$, at the $\Gamma$ point.

\begin{tabular}{cccccc}
\hline A-point & L-point & M-point & Г-point & H-point & K-point \\
\hline 16.391 & 18.583 & 19.456 & 18.743 & 16.391 & 19.335 \\
16.391 & 18.583 & 18.526 & 16.979 & 16.391 & 19.335 \\
15.608 & 18.105 & 18.309 & 16.979 & 15.608 & 18.187 \\
15.608 & 18.105 & 17.429 & 15.253 & 15.608 & 18.187 \\
15.608 & 17.114 & 16.449 & 14.441 & 15.608 & 14.577 \\
15.608 & 17.114 & 15.723 & 14.441 & 15.608 & 14.577 \\
11.646 & 10.815 & 13.250 & 12.515 & 11.646 & 13.667 \\
11.646 & 10.815 & 11.474 & 10.296 & 11.646 & 13.667 \\
-0.632 & -1.998 & -1.082 & 0 & -0.632 & -1.574 \\
-0.632 & -1.998 & -2.242 & 0 & -0.632 & -1.574 \\
-0.632 & -2.107 & -2.946 & -1.187 & -0.632 & -4.646 \\
-0.632 & -2.107 & -3.626 & -1.187 & -0.632 & -4.646 \\
-3.543 & -5.700 & -4.249 & -1.187 & -3.543 & -4.674 \\
-3.543 & -5.700 & -6.039 & -6.446 & -3.543 & -4.674 \\
-17.951 & -16.7 & -16.424 & -16.904 & -17.951 & -16.515 \\
-17.951 & -16.7 & -16.956 & -18.733 & -17.951 & -16.515 \\
\hline
\end{tabular}


further comparison of our findings with some future, experimental data. Such experiments can include optical transitions. X-ray and UV spectroscopy measurements can provide the widths of upper and lower groups of valence bands and the total width of the entire valence band.

Effective masses are used in determining transport properties of a system, i.e., the transport of electrons under the influence of electric fields or carrier gradients. Agreements between measured and calculated electron effective masses show the accuracy of the shape and curvature of the calculated band. Our calculated electron effective mass and those of the light hole and heavy holes 1 and 2, from $\Gamma$ to $A$, are respectively $1.298,-0.360,-2.107$ to -2.685 , and -2.107 to $-2.68 \mathrm{~m}_{\mathrm{o}}$. We are not aware of measured values of these effective masses for w-BeO.

\section{Discussion}

The content of Table 2, as opposed to our calculated band gap of $10.30 \mathrm{eV}$, raises the question as to possible reasons for the serious underestimation by the previous ab-initio calculations. From the description of our method, it is apparent that heeding the second DFT theorem requires an energy minimization far beyond the linear minimization attainment with self-consistent iterations using a single basis set. We have not seen any indication, in the pertinent articles, that any of the nine (9) ab-initio LDA and eleven (11) ab-initio GGA calculations in Table 2 employed successively larger basis sets to reach verifiably the ground state of the system. Consequently, their respective results of $7.0-7.8 \mathrm{eV}$ and $6.99-9.50 \mathrm{eV}$, for the band gap of wurtzite $\mathrm{BeO}$, should not be expected to agree with experiment, given that they do not possess the full, physical content of DFT.

Previous ab-initio calculations, with single basis sets, led to stationary solutions. There is an infinite number of stationary solutions. Some do not obtain the ground state, due to deficiency in their basis sets. Others may produce the absolute minima of the occupied energies (the ground state), however, practically all of them have over-complete basis sets that lead to unphysical lowering of unoccupied energies, resulting in an underestimation of the band gaps. The chances for a single basis set calculation to produce the absolute minima of the occupied energies (i.e., the ground state), without using a basis set that is over-complete for the description of the ground state, are practically zero. The use of successively larger basis sets in consecutive, self-consistent calculations is the only way we know 1) to ensure the attainment of the absolute minima of the occupied energies (the ground state) and 2) to avoid unnecessarily large basis sets. Such large basis sets are over-complete and they unphysically lower unoccupied energies to result in band gaps smaller than the actual DFT and experimental ones.

The above points also explain the reason that our estimated band gap is in excellent agreement with experiment, i.e., our computations strictly adhered to 
conditions inherent to the validity of DFT. The successively larger basis sets led to a minimization far beyond what is attainable with a single basis set following self-consistency iterations. Even though the self-interaction correction (SIC) calculation with a hybrid potential, in Table 2, led to agreement with experiment, these efforts do not resolve the underestimation problem due to the ad hoc nature of the implementation of SIC and of a hybrid functional potentials [40]. In fact, the corresponding potentials are not entirely DFT potentials, as they cannot be obtained, from first principle, as functional derivatives of ab-initio exchange correlation energies.

\section{Conclusions}

We have described the ground state electronic and related properties of $\mathrm{w}-\mathrm{BeO}$ by using the BZW-EF method. It ensures the full physical content of calculated quantities by adhering to the pertinent DFT theorem. Unlike previous ab-initio calculations, our calculated energy gap of $10.30 \mathrm{eV}$ is in agreement with experiment. Our calculated density of states (DOS) and partial density of states (p-DOS) are in good agreement with results from electron momentum spectroscopy (EMS). We expect future measurements to confirm our findings. To the best of our knowledge, no measurements of the electron effective masses are available for comparison with our calculated ones.

Our findings for $\mathrm{w}-\mathrm{BeO}$ illustrate the effectiveness of the BZW-EF method to describe accurately electronic and related properties of semiconductors and insulators, using DFT potentials. We recall that the method: 1) searches and obtains the absolute minima of the occupied energies, and 2) avoids very large basis sets that are over-complete for the description of the ground state. The second DFT theorem requires this search. Hence, future DFT calculations should search for and attain verifiably the absolute minima of the occupied energies of systems under study, and avoid over-complete basis-sets, in order to produce results with the full, physical content of DFT and that agree with experiment.

\section{Acknowledgements}

This research work was funded in part by the US Department of Energy, National, and Nuclear Security Administration (NNSA) (Award Nos. DE-NA0001861 and DE-NA0002630), the National Science Foundation (NSF HRD-1002541), LaSPACE, and LONI-SUBR.

\section{References}

[1] Roessler, D.M., Walker, W.C. and Loh, E. (1960) Journal Physics Chemistry Solids, 30, 157-167.

[2] Freeouf, J.L. (1960) Physical Review, 7, 3810-3830.

[3] Peterson, C.W. and Palma, G.E. (1973) Journal of Optical Society Of America, 63, 387-388. 
[4] Bellamy, B., Baker, T.W. and Livey, T.W. (1962) Journal of Nuclear Materials, 6, 1-4.

[5] Grundler, R., Breuer, K. and Tews, W. (1978) Physics Statics of Solids, 86, 329-338.

[6] Chang, K.J., Froven, S. and Cohen, M.L. (1983) Journal of Solid State Physics, 16, 3475-3480.

[7] Mazurenko, V.V., Rudenko, A.N., Kvashin, Y.O., Mazurenko, V.G., Novoselov, Y.N., Pusto-varoy, V.A., Kukharenko, V.A. and Cholakh, S.O. (2011) Journal of Experimental and Theoretical Physics, 112, 877-883.

[8] Baumeier, B., Kruger, P. and Pollman, J. (2007) Physical Review B, 76, 1-8.

[9] Yu, B.R., Yang, J.W., Guo, H.Z., Ji, G.F and Chen, X.R. (2009) Physical Review B, 404, 1940-1946.

[10] Duan, Y., Shin, H. and Qin, L. (2008) Physics Letters A, 372, 2930-2933.

[11] Xu, Y.N. and Ching, W.Y. (1993) Physical Review B, 48, 4335-4351.

[12] Chang, K.J. and Cohen, M.L. (1984) Solid State Communications, 50, 487-491.

[13] Vydrov, O.A. (2007) Correction the Self-Interaction Error of Approximate Density Functionals. Doctoral Dissertations, Rice University Database, 21-25.

[14] Guo, Z.C., Luo, F., Ji, G.F., Cai, L.C. and Cheng, Y. (2014) Physica B, 438, 60-64.

[15] Xu, Q., Zhang, X.W., Fan, W.J., Li, S.S. and Xia, J.B. (2008) Computational Material Science, 44, 72-78.

[16] Dong, L. and Alpay, S.P. (2011) Physical Review B, 84, 1-7.

[17] Duman, S., Sutlu, A., Bagci, S., Tutuncu, H.M. and Srivastava, G.P. (2009) Journal of Applied Physics, 105, 1-8.

[18] Marnetto, A., Penna, M., Bertazzi, F., Belloti, E. and Goano, M. (2008) Optical Quantum Electron, 40, 1135-1141.

[19] Milman, V. and Warren, M.C. (2001) Journal of Condense Matter, 13, 241-251.

[20] McLeod, J.A., Wilks, R.G., Skorikov, N.A., Finkelstein, L.D., Samak, M.A., Kurmaev, E.Z. and Moewes, A. (2010) Physical Review B, 81, 1-9.

[21] Amrani, B., Hassan, F.E.H. and Akbarzadeh, H. (2007) Journal of Physics: Condensed Matter Solids State Physics, 19, 1-12.

[22] Groh, D., Pandey, R., Sahariah, M.B., Amzallag, E., Bareille, I. and Rerat, M. (2009) Journal of Physics and Chemistry of Solids, 70, 789-795.

[23] Shein, I.R., Kiiko, V.S., Makurin, Y.N., Gorbunova, M.A. and Ivanovskii, A.L. (2007) Physics of Solid State, 49, 1067-1073.

[24] Qi-Li, Z., Ping, Z., Feng, S.H. and Feng, L.H. (2008) Chinese Physics, 17, 1341-1348.

[25] Lichanot, A., Chaillet, M. and Larrieux, C. (1992) Chemical Physics, 164, 383-394.

[26] Kaambre, T., Kikas, A., Kooser, K., Kisand, V., Kim, M., Saar, A., Nommiste, E., Ivanov, V., Pustovarow, V. and Martin-Son, I. (2007) Journal of Electron Spectroscopy and Related Phenomena, 156-158, 299-302.

[27] Camp, P.E.V. and Oren, V.E.V. (1996) Journal of Physics. Condense Matter, 8, 3385-3390.

[28] Li, D., Zhang, P. and Yan, J. (2014) Scientific Reports, 1-5.

[29] Ren, L., Cheng, L., Feng, Y. and Wang, X. (2012) Journal of Chemical Physics, 137, 1-5.

[30] Boettger, J.C., Honnell, K.G., Mori, Y., Niiya, N. and Mizuno, T. (2006) AIP Conference Proceedings, 845, 33-36. 
[31] Duvarney, R.C., Garrison, A.K. and Thorland, R.H. (1969) Physical Review, 188, 657-661.

[32] Loh, E. (1968) Physical Review, 166, 673-678.

[33] Ivanov, V., Kirm, M., Pustovarov, V. and Kruzloy, A. (2006) Radiation Measurements, 42, 742-745.

[34] Sashin, V.A., Dorsett, H.E., Bolorizadeh, M.A. and Ford, M.J. (2000) Journal of Chemical Physics, 113, 8175-8182.

[35] Argoitia, A., Angus, J.C., Wang, L., Ning, X.I. and Pirouz, P. (1993) Journal of Applied Physics, 73, 4305-4312.

[36] Reinberg, A.R. (1964) Journal of Chemical Physics, 41, 850-855.

[37] Downs, J.W., Ross, F.K. and Gibbs, G.V. (1985) Acta Crysta, 4, 425-431.

[38] Yum, H.J., Akyol, T., Lei, M., Hudnall, T., Bersuker, G., Downer, M., Bielawski, C.W., Lee, J.C. and Banerjee, S.K. (2011) Journal of Applied Physics, 109, 1-4.

[39] Ivanovskii, I.R., Shein, A.L., Makurin, Y.N., Kiiko, V.S. and Gorbunova, M.A. (2009) Inorganic Materials, 45, 223-234.

[40] Bagayoko, D. (2014) AIP Advances, 4, Article ID: 127104.

[41] Bagayoko, D., Zhao, G.L., Fan, J.D. and Wang, J.T. (1998) Journal of Physics. Condens Matter, 10, 5645.

[42] Zhao, G.L., Bagayoko, D. and Williams, T.D. (1998) Physical Review B, 60, 1563-1572.

[43] Ekuma, C.E., Bagayoko, D., Jarell, M. and Moreno, J. (2012) AIP Advances, 2, Article ID: 032163.

[44] Bagayoko, D., Franklin, L. and Zhao, G.L. (2004) Journal of Applied Physics, 96, 4297-4301.

[45] Jin, H., Zhao, G.L. and Bagayoko, D. (2007) Journal of Applied Physics, 101, Article ID: 0333123.

[46] Bagayoko, D., Franklin, L., Zhao, G.L. and Jin, H. (2008) Journal of Applied Physics, 103, Article ID: 096101.

[47] Bagaoko, D. and Franklin, L. (2005) Journal of Applied Physics, 97, Article ID: 123708.

[48] Ekuma, C.E., Jarrell, M., Moreno, J. and Bagayoko, D. (2013) Physics Letter A, 337, 2172-2176.

[49] Zhao, G.L. and Bagayoko, D. (2000) New Journal of Physics, 2, 1.

[50] Ceperley, D.M. and Alder, B.J. (1980) Physical Review Letter, 45566.

[51] Vosko, S.H., Wilk, L. and Nusair, M. (1980) Canadian Journal of Physics, 58, 1200.

[52] Ekuma, C.E., Jarell, M., Moreno, J., Franklin, L., Zhao, G.L., Wang, J.T. and Bagaoko, D. (2012) Material Chemistry and Physics, 136, 1137-1142.

[53] Franklin, L., Ekuma, C.E., Zhao, G.L. and Bagayoko, D. (2013) Journal of Physics and Chemistry of Solids, 74, 729-736.

[54] Sahin, V.A., Bolorizadeh, M.A., Kheifets, A. and Ford, M.J. (2007) Electronics Band Structure of Beryllium Oxide PACS Number: 71.20.PS.P:1-77. 